\title{
XI. - VALEUR ALIMENTAIRE CHEZ LA POULE PONDEUSE
}

\author{
J. MOAL $\left({ }^{1}\right)$ et C. CALET $\left({ }^{2}\right)$ \\ avec la collaboration technique de J. Castaing, R. Gabastov, Ph. Dascon (1) J. M. Regmier (3)
}

(1) Association générale des Producteurs de maīs, 64-Pau

(2) Station de Recherches avicoles, Institut national de la Recherche agronomique, 37-Nouzilly.

(3) Centre national d'Étude et d'Expérimentation du Machinisms agricole. Parc de Tourvoie, 92-Antony.

\section{RÉSUMÉ}

360 poules $\left(\mathrm{M}_{4} \mathrm{I}\right)$ ont été alimentées ad libitum dès leur entrée en ponte, pendant 280 jours. Les régimes contenaient l'un des six échantillons de maïs précédemment définis, à raison de 69 p. Ioo.

En aucun cas les échantillons de maïs ne se sont révélés toxiques. Au contraire, l'effet principal de la conservation avant le séchage $(E$ et $F)$ a été l'augmentation significative de la consommation quotidienne $(3,4$ et $5,8 \mathrm{p}$. Ioo respectivement), d'emblée pour les mais $\mathrm{F}$, un peu plus tard pour les maïs E. Pour ces derniers, la consommation supplémentaire s'accompagne d'une ponte plus intense, sans que l'indice de consommation ne soit modifié. En revanche, les maïs $F$ n'ont pas permis une amélioration de la ponte : leur efficacité reste donc inférieure de 6 p. Ioo par rapport aux traitements $\mathrm{R}$ pris comme témoins.

$L^{\prime}$ effet de la température de séchage n'est pas significatif, mais un séchage à $\mathrm{I}_{40^{\circ} \mathrm{C}}$ tend à diminuer légèrement la consommation quotidienne et la ponte; il tend à fournir des œufs un peu plus lourds.

\section{I. - INTRODUCTION}

L'étude de l'influence de divers traitements de récolte et de conservation du maïs a fait 1'objet de quelques travaux chez le poussin (CALET et al., Ig6o, Ig66 ; EMERICK et al., I96r) mais nous n'avons pas eu connaissance de travaux analogues chez la poule pondeuse. Or, la longue durée des essais permet de mettre en évidence des effets à long terme, par exemple un accumulation d'éventuelles toxines. Par ailleurs, la poule est une animal assez sensible, capable d'extérioriser les carences frustres de son régime. Enfin et surtout, beaucoup d'aviculteurs se plaignent d'accidents survenus en cours de ponte par l'introduction, dans le régime alimentaire d'un mais qu'ils considèrent " mauvais".

Aussi avons-nous alimenté des poules avec des régimes contenant $69 \mathrm{p}$. roo de l'un des 6 échantillons de maïs décrits plus haut. 


\section{II. - MATÉRIEL ET MÉTHODES}

360 poules $\mathrm{M}_{4} \mathrm{I}$ âgées de $\mathrm{I} 6$ semaines sont placées en cages individuelles au début de l'été dans un poulailler sans fenêtre. Elles sont éclairées pendant I 2 heures chaque jour. Cet éclairage augmente de 15 minutes par semaine jusqu'à un plafond de 15 heures.

Les 360 sujets sont choisis parmi 6oo poulettes dont on avait contrôlé précédemment le gain de poids à 4 et $\mathbf{r} 2$ semaines d'âge. On sait en effet que la croissance des poulettes retentit directement sur leurs performances de ponte à l'âge adulte (MOAL et CASTAING, r970) a et b. Aussi les poulettes sont réparties en six classes de poids et, dans chacun de ces groupes, ro répétitions de chacun des régimes sont constituées. Ainsi 60 poules de chaque lot reçoivent leur régime ad libitum.

\section{TABLEAU I}

Formule et caracteristiques principales du régime expérimental

\begin{tabular}{|c|c|c|c|}
\hline \multicolumn{2}{|l|}{ Formule : p. 100} & \multicolumn{2}{|l|}{ Caractéristiques } \\
\hline Matières premières & & & \\
\hline 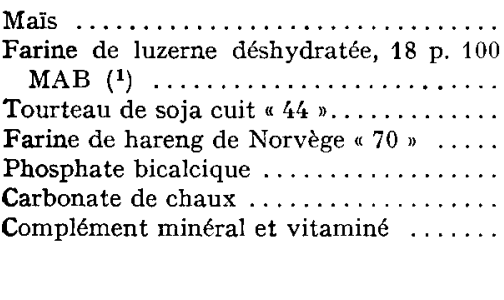 & $\begin{array}{l}3,5 \\
15 \\
3 \\
1,5 \\
6 \\
2 \\
100\end{array}$ & 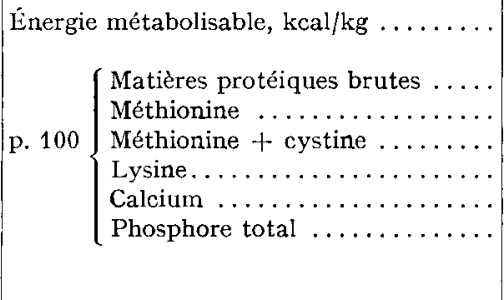 & $\begin{array}{l}2880 \\
15,7 \\
0,322 \\
0,598 \\
0,598 \\
3,12 \\
0,75\end{array}$ \\
\hline
\end{tabular}

(1) Matière azotée brute.

Les six régimes expérimentaux ont des caractéristiques correspondant aux normes alimentaires en vigueur (tabl. I). Ils ne different que par la nature du maïs.

L'essai débute lorsque le troupeau a pondu à $5 \mathrm{p}$. Ioo. Il se déroule pendant ro périodes de 28 jours. Les cufs sont ramassés chaque jour et pesés chaque semaine; la consommation est contrôlée tous les 28 jours.

\section{III. - RÉSULTATS}

Les principaux résultats sont rassemblés dans le tableau 2. Seuls les résultats par facteur seront commentés, puisque l'analyse statistique ne révèle aucune interaction significative entre les facteurs étudiés.

\section{I. - Effet de la température de séchage}

L'effet de la température de séchage est apparemment négligeable. L'examen des résultats nous amène cependant à faire état des nuances suivantes :

- Si la consommation par jour et le nombre d'cufs pondus sont somme toute assez voisins, la différence va toujours dans le même sens, en faveur d'un séchage 
à $80^{\circ} \mathrm{C}$; cette différence est même significative pour le second critère au cours de la $5^{\mathrm{e}}$ période de ponte $(2 \mathrm{I}, \mathrm{O}$ œufs contre $20, \mathrm{I})$.

- A l'opposé, le poids moyen de l'œuf est systématiquement plus élevé chez les poules ayant reçu du maïs séché à $140^{\circ} \mathrm{C}$, les écarts restant dans l'ensemble, il est vrai, extrêmement faibles.

- Les rapports entre la consommation et la ponte (consommation par œuf ou par kilogramme d'œufs) ne permettent pas de dégager une quelconque tendance dans un sens ou dans l'autre.

\section{TABLEAU 2}

Influence des traitements de séchage et de conservation d'un mais sur les principales performances de poules pondeuses

Durée de l'expérience : ro périodes de 28 jours

\begin{tabular}{|c|c|c|c|c|c|c|c|c|}
\hline & \multirow[b]{2}{*}{$\begin{array}{c}\mathrm{CV}(\mathbf{1}) \\
(\%)\end{array}$} & \multicolumn{3}{|c|}{ Température de séchage } & \multicolumn{4}{|c|}{ Méthode de préstockage } \\
\hline & & $80^{\circ} \mathrm{C}$ & $1_{1}^{\prime} 0^{\circ} \mathrm{C}$ & $\begin{array}{l}\text { Signifi- } \\
\text { cation }\left({ }^{2}\right)\end{array}$ & $\mathrm{R}$ & $\mathrm{E}$ & $\mathrm{F}$ & $\begin{array}{l}\text { Signifi- } \\
\text { cation et } \\
\text { p.p.d.s. }\left(^{2}\right)\end{array}$ \\
\hline Consommation ....... & 8,9 & 112,3 & 111,6 & NS & $108,7^{b}$ & $112,2^{a}$ & $115,0^{a}$ & $2,9 * *$ \\
\hline Nbre d'œufs pondus $\left({ }^{3}\right)$ & 13,0 & 179,3 & 175,9 & NS & 175,9 & 180,6 & 176,3 & NS \\
\hline Poids moyen de l'cuf,g & 5,0 & 60,8 & 61,0 & NS & 60,8 & 61,0 & 61,1 & NS \\
\hline $\begin{array}{l}\text { Consom. par œuf, g .. } \\
\text { Consom. par kg d'œufs }\end{array}$ & 18,4 & 160,0 & 163,7 & NS & $158,1^{a}$ & $158,7^{a}$ & $168,7^{b}$ & $8,7^{*}$ \\
\hline$g \quad \ldots \ldots \ldots \ldots$ & 18,7 & 2632 & 2683 & NS & $2602^{a}$ & $2607^{a}$ & $2764^{b}$ & $146^{*}$ \\
\hline
\end{tabular}

(1) Coefficient de variation.

(2) P.p.d.s. = plus petite différence significative, au seuil de 5 p. 100.

NS $=$ non significatif.

$*$ = significatif au seuil de 5 p. $100 ;{ }^{* *}=$ significatif au seuil de 1 p. 100.

Les résultats accompagnés de lettres différentes sont significativement différents.

(3) A l'exclusion des cufs non commercialisables (mous ou à coquille imparfaite).

\section{2. - Effet de la méthode de préstockage}

L'examen des résultats obtenus met en lumière un effet très net des méthodes de conservation sur la consommation quotidienne : elle est sensiblement supérieure chez les poules ayant reçu des maïs ayant attendu avant le séchage ( $\mathrm{E}$ et $\mathrm{F}$ ).

Le comportement alimentaire des poules a d'ailleurs varié au cours de 1'essai, comme le montre la figure I :

- d'emblée, la consommation des maïs F est supérieure à celle des maïs témoins (R) ;

- celle des maïs E est aussi, la plupart du temps, supérieure à celle constatée pour les maïs $\mathrm{R}$, mais elle oscille jusqu'à la $5^{\mathrm{e}}$ période entre les valeurs constatées avec les deux autres maïs pour ensuite se rapprocher définitivement de $F$, sans jamais l'atteindre. 
Les résultats ne font pas apparaitre de différence significative entre les taux de ponte ainsi qu'entre les poids moyens des ceufs.

L'indice de consommation (consommation par ceuf ou par kilogramme d'cufs pondus) montre que seuls les maïs F sont défavorisés. Dans ce cas la consommation supérieure ne s'accompagne pas d'une meilleure performance. En revanche, avec le maïs $\mathrm{E}$, la consommation accrue d'aliment (3,5 grammes/jour de plus que les témoins " $\mathrm{R} 川)$ est associée à une ponte meilleure ( $+4,7$ œufs sur tout l'essai).

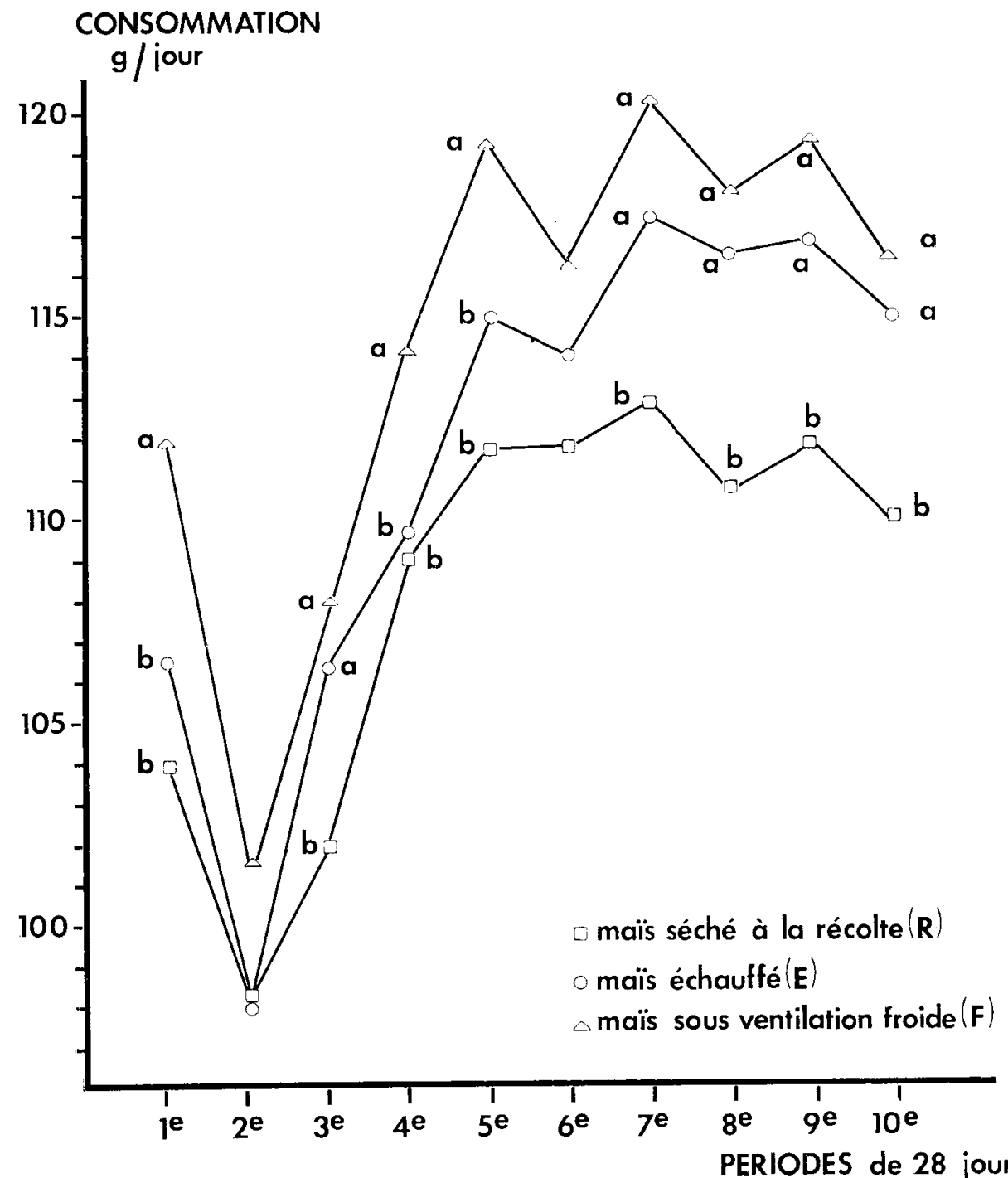

FIG. I. - Infiuence du mode de préstockage du mais sur la consommation de la poule pondeuse.

a, b : Les moyennes affectées des mêmes lettres ne diffèrent pas significativement. 


\section{IV. - DISCUSSION}

Il faut tout d'abord noter qu'aucun effet toxique n'a été enregistré à la suite de l'ingestion des maîs $\mathrm{E}$ et $\mathrm{F}$. La mortalité au cours de l'essai $(8,3$ p. Ioo) est celle qu'on enregistre habituellement. Elle était sensiblement la même dans tous les lots. Or la poule pondeuse est très sensible à différentes toxines fongiques comme l'aflatoxine qui provoque une chute de la ponte et la dégénérescence graisseuse du foie (DE ANDREs et al. I968). Ici la mortalité était due essentiellement à des leucoses viscérales ou à des paralysies de Marek. On peut donc affirmer que les échantillons de maïs échauffés (E) ou conservés sous ventilation d'air refroidi (F) ne possédaient pas d'aflatoxine, dans les conditions de nos essais.

En revanche, contrairement à toute attente, les lots stockés avant séchage ont eu un effet favorable sur l'appétit de la poule puisque la consommation quotidienne de ces dernières s'est élevée respectivement de 3,4 et $5,8 \mathrm{p}$. Ioo pour les lots $\mathrm{E}$ et $\mathrm{F}$ au-dessus de la consommation moyenne du lot témoin ( $\mathrm{R}$ ).

Pour les mais $F$, cet effet positif ne se traduit malheureusement par aucun bénéfice sur les performances de ponte, à l'inverse des maìs $\mathrm{E}$, dont l'efficacité se montre équivalente à celle des témoins.

\section{V. - CONCLUSION}

Le stockage du maïs avant le séchage n'aboutit pas forcément à la formation de toxines dans le grain puisque un essai de longue durée chez la poule pondeuse (280 jours d'expérience) n'a pas permis de déceler, dans les conditions de nos essais, d'effets toxiques quelconques. Il n'a pas entraîné non plus de performances mauvaises. Bien au contraire les diverses méthodes de conservation du maïs avant son séchage se sont traduites par une augmentation de l'appétit qui s'est parfois accompagné d'une amélioration non significative du nombre des œufs pondus.

\section{SUMMARY}

\section{XI. - FEEDING VALUE OF MAIZE IN THE I,AXING HEN}

260 hens (M $4 \mathrm{I}$ ) were fed ad libitum for 280 days after the beginning of the laying period. The diets contained $69 \mathrm{p}$. Ioo of one of the six previously described maize samples.

The maize samples have no toxic effect. On the contrary, the main action of the conservation before drying ( $\mathrm{E}$ and $\mathrm{F}$ ) was a significant increase in the daily food intake ( 3.4 and $5.8 \mathrm{p}$. roo respectively), which was immediate for the $\mathrm{F}$ samples and a little later for the $\mathrm{E}$ samples. When using these samples (E), the increased food intake was accompanied by a more intense egg laying, without modification of the food conversion ratio. On the other hand, the F maize samples did not improve the laying performances; thus, the food efficiency of this maize is lower $(6 \mathrm{p}$. Ioo) than that obtained with the control treatments (R).

The effect of the drying temperature is not significant, but drying at $140^{\circ} \mathrm{C}$ tends to decrease, but only a little, the daily food intake and laying; however, the eggs obtained seem to be slightly heavier. 


\section{RÉFÉRENCES BIBLIOGRAPHIQUES}

DE ANDRes V., DE ANDRes Juana, CALET C., I968. Modifications de la composition biochimique des tissus de la poule sous l'effet de doses subcliniques d'Aflatoxine dans la nourriture. C. R. III $d$ European Poultry Conference, Jérusalem, 35r-356.

Calet C., de Lambilly H., I960. Étude de la valeur alimentaire du maïs séché artificiellement pour la croissance du poussin. I. Influence du mode de séchage sur la disponibilité des acides aminés. Ann. Zootech., 9, I8I-I84.

CALet C., TARdif H., I96o. Étude de la valeur alimentaire du maīs séché artificiellement pour la croissance du poussin. II. Influence de la durée qui sépare la récolte du séchage. Ann. Zootech., 9, 349-354.

Emerik R. J., Carlson C. W., Winterfeld H. L., I96I. Effect of Heat Drying upon the Nutritive Value of Corn. Poult. Sci., 40, 99 I-994.

Moal J., Castaing J., I97o. a) Influence de la batterie et du passé en poussinière sur quelques performances de poules pondeuses. Nouvelles de l'Aviculture, 128, 9-Io.

Moal J., Castaing J., I970. b) Poids des poulettes et performances en ponte. Nouvelles de l'A viculture, 129,9 -Io. 